\title{
INVESTIGATION ON DEPTH OF HEAT AFFECTED ZONE OF DISCHARGE SPOT BY HIGH REPETITIVE RATE YAG LASER-INDUCED DISCHARGE TEXTURING
}

\author{
ZHENGYANG LI*, ${ }^{*}$, MINGJIANG YANG ${ }^{\dagger}$, MINLIN ZHONG* and WENJIN LIU* \\ *Key Laboratory for Advanced Manufacturing by Materials Processing Technology, \\ Department of Mechanical Engineering, Tsinghua University, \\ Beijing 100084, P. R. China \\ ${ }^{\dagger}$ Institute of Mechanics, Chinese Academy of Sciences, \\ Beijing 100080, P. R. China
}

Received 5 December 2005

\begin{abstract}
It is known that the press formability and the elongation of laser textured sheet are improved, and the service life of textured roll is longer than that of the un-textured roll due to hardening of the treated surface. One of the goals to develop high repetitive rate YAG laser-induced discharge texturing (LIDT) is to get deeper hardening zone. By observing and measuring cross-section of LIDT spots in different discharge conditions, it is found that the single-crater, which is formed by the discharge conditions of anode, which is covered by an oil film and with rectangular current waveform, has the most depth of heat affected zone (HAZ) comparing with other crater shapes when discharge energy is the same. The depth of HAZ is mainly depends on pulse duration when the discharge spot is single-crater. The results are analyzed.
\end{abstract}

Keywords: Laser; heat affected zone; discharge.

\section{Introduction}

The surface of steel sheet used in automobiles is normally treated to provide a matte finish to give a proper surface roughness for press formability and to get high image clarity for superior quality. ${ }^{1,2}$ The roll is usually textured by various texturing techniques, such as electrical discharge texturing (EDT), laser texturing (LT) or electronic beam texturing (EBT), and then it rolls sheets. Investigation show that while the press formability and the elongation of the sheet are improved, the service life of textured roll is also longer than that of un-textured roll due to hardening of the treated surface, i.e., resolidification and transformation of the radiated area. ${ }^{3,4}$ Former researches are mainly focused on the pattern and the shape of crater, i.e., the radiated area, little attention is paid to the effect of hardening zone of crater and its attribution to the service life of roll. To improve service life of roll under the heavy load working conditions, it is necessary to deepen the hardening zone.

Since the absorptivity of laser by metal is limited, the depth of hardening zone by laser texturing is relatively low. For instance, it is only several dozens of micrometers for YAG laser texturing. To deepen hardening zone while the deterministic crater pattern is expected, it is necessary to increase the pulse energy and the density of pulse power. Increasing laser power is one of the solutions, but the repetitive rate of laser pulse has to be decreased. Though EDT may deepen hardening zone, its crater pattern is random. The crater pattern of EBT is deterministic and the absorptivity of electronic beam by metal is high, but the processing must be carried out in the vacuum. 
To take advantages of the controllability of laser and the high electrical absorptivity by metal, an innovative texturing technique, high repetitive rate YAG laser-induced discharge texturing (LIDT), is developed. We have presented the crater morphology of LIDT, i.e., the patterns and shape of crater, on several materials with different media, discharge waveforms and electrical polarities in Ref. 5. Due to resolidification and transformation of the radiated area, a hardening zone is formed under the LIDT spot. Our main concern is the depth of hardening zone. The present work focuses on the depth variation with various parameters in the processing. The results are illustrated and analyzed.

\section{Experiments}

When an electrical pulse applies on the two electrodes in air, whose clearance is approximately $0.5 \mathrm{~mm}$, no breakdown will occur if the pulse peak is about several hundred volts. If a laser pulse synchronized with electrical pulse radiates on one of two electrodes, a plasma plume appears, that serves as discharge channel and the breakdown may occur between the electrodes far below the breakdown threshold. The duration of electrical pulse is far longer than that of the laser pulse. The discharge is triggered by laser pulse and is shut off by the electrical pulse that is controlled by IGBT (insulated gate bipolar transistor) of the power supply. If the roll is one of the two electrodes and a conical metal rod is the other, discharge crater will be distributed on the roll by a serial laser-induced discharge with the roll rotating and the moving of laser set and the conical metal rod along the axial of the roll (Fig. 1).

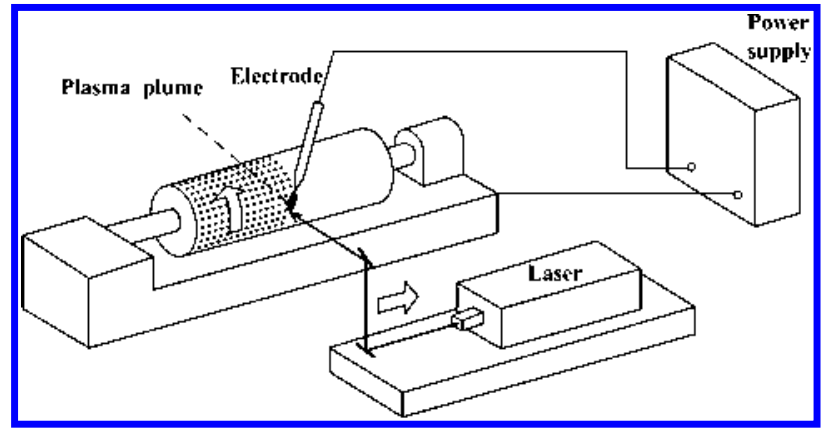

Fig. 1. The principle of high repetitive rate YAG laserinduced discharge texturing (LIDT).
With hardware and software controlling, deterministic or random crater patterns can be obtained easily. ${ }^{5}$

The experiment was carried out in air, but the surface of roll might be covered by a thin oil film sometimes, which meant that the discharge media between the two electrodes were either air or air and oil. The oil was engine oil and the thickness of oil film was about half clearance between the two electrodes. The tip angle of conical electrode was $20^{\circ}$ $45^{\circ}$. The rolls were made of steel (AISI 1045) and cast iron, respectively, and the conical electrode was made of $\mathrm{CuW}$. The clearance between the two electrodes was $200-300 \mu \mathrm{m}$. The laser pulse was modified by a Q-switch on a 150 watts Nd: YAG laser oscillator. For safety reasons, the roll was always connected to the ground, but the electrical polarities between the roll and the conical electrode were alternated that mean that the roll could be either cathode or anode. The current waveform was controlled by pulse duration and capacity voltage. Figure 2 showed current waveforms with pulse duration being set to $300 \mu$ s and capacity voltage varied from 50 to $150 \mathrm{~V}$. Figures 3 and 4 show that current peak was mainly controlled by capacity voltage if the pulse duration was not too long and triangular and rectangular waveforms could be obtained by adjusting parameters of power supply. It should be noticed that the real discharge

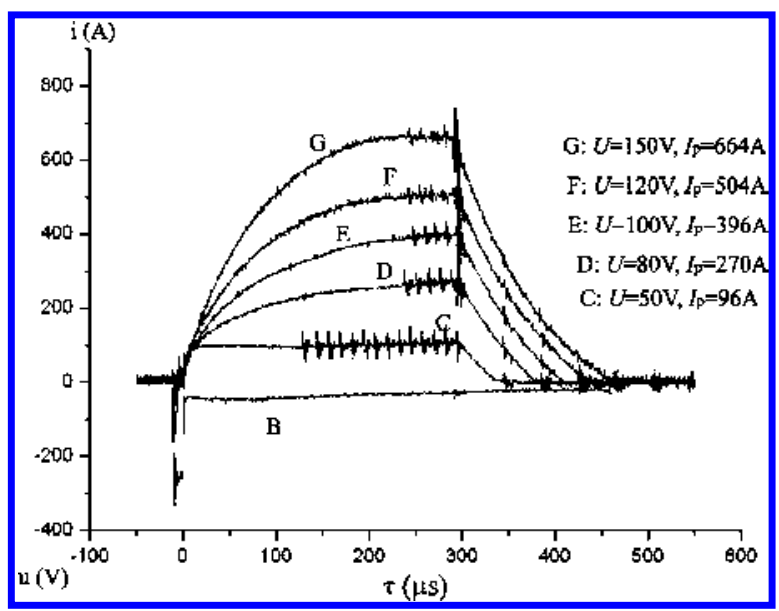

Fig. 2. Current waveforms at $300 \mu$ s pulse duration with different capacity voltage. $B$ is voltage waveform, $C, D$, $E, F, G$ are current waveforms. $U$ means capacity voltage. $I_{\mathrm{p}}$ is the corresponding current peak.

*High negative volts $(300-350 \mathrm{~V})$ at $\tau=0$ is the voltage applied before breakdown. 
duration of triangular waveform was about double of the setting (Fig. 3) and voltage waveforms were maintained constant after discharging (curve $B$ at Figs. 2-4) which was a distinguishing feature of arc discharge.

To investigate the relationship of the depth of heat affected zone (HAZ) of a LIDT spot with different current waveforms and discharge energy $E$, two serial experiments were designed. It was known that there were two types of crater shape, namely, single-crater and multi-crater. ${ }^{5}$ For the former, the discharge waveforms were the triangular and the rectangular. For the latter, to compare the result of different discharge waveforms, the energy of each discharge pulse was set to be

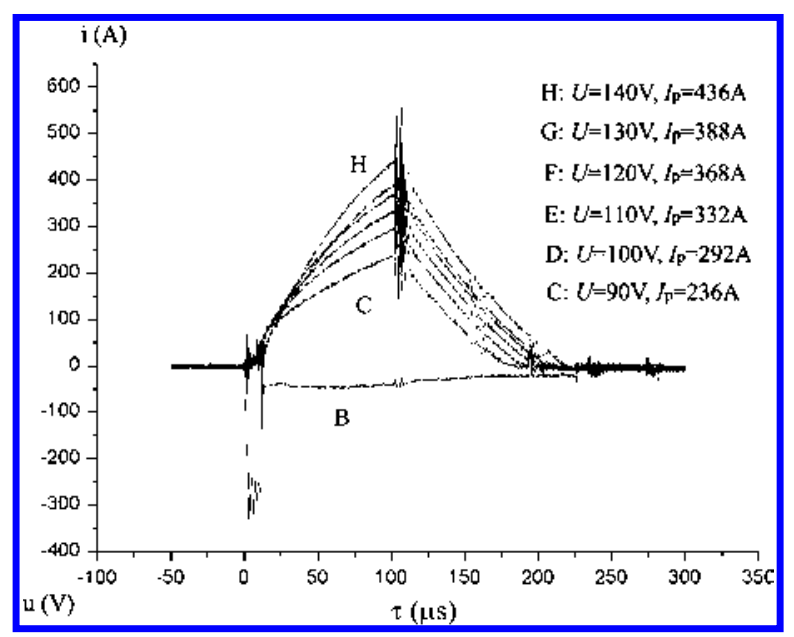

Fig. 3. Current waveforms at $100 \mu$ s pulse duration with different capacity voltage. $B$ is voltage waveform, from down $C$ to up $H$ are current waveforms. $U$ means capacity voltage. $I_{\mathrm{p}}$ is the corresponding current peak.

*High negative volts $(300-350 \mathrm{~V})$ at $\tau=0$ is the voltage applied before breakdown. the same as $1 \mathrm{~J} . E=\int_{0}^{\tau} i u \mathrm{dt}$, where $i$ and $u$ are current and voltage, respectively, were recorded by Tektronix TDS 210 oscillograph, $\tau$ was pulse duration.

The specimens were cut from the roll along the edge of discharge spots and were inlaid in transparent material, so that the cross-section of spots was the plane being grinded (Fig. 5). If the spots were along a line which is parallel to the plane, we could grind the plane step by step to the center of the spots by watching through transparent material. It was assumed that the cross-section profiles of crater, melt zone (MZ) and HAZ of a discharge spot were dome, and defined $h_{1}, h_{2}$ and $h_{3}$ as the depth of crater, MZ and HAZ, respectively, then the maximum $h_{3}$, i.e., $h_{3 \max }$,

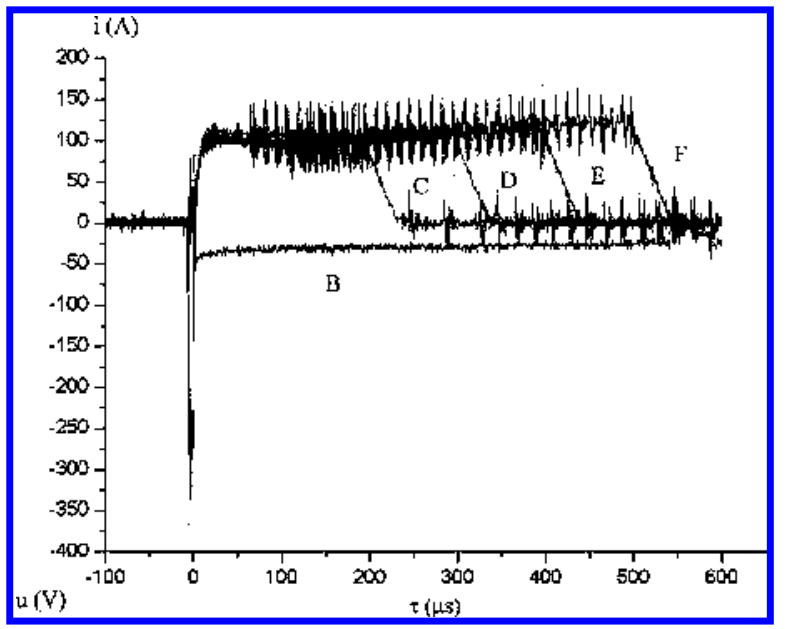

Fig. 4. Current waveforms at capacity voltage $50 \mathrm{~V}$ with different pulse durations. $B$ is voltage waveform. $C, D, E$ and $F$ are current waveforms with pulse duration $200 \mu \mathrm{s}$, $300 \mu \mathrm{s}, 400 \mu \mathrm{s}, 500 \mu \mathrm{s}$, respectively.

${ }^{*}$ High negative volts $(300-350 \mathrm{~V})$ at $\tau=0$ is the voltage applied before breakdown.

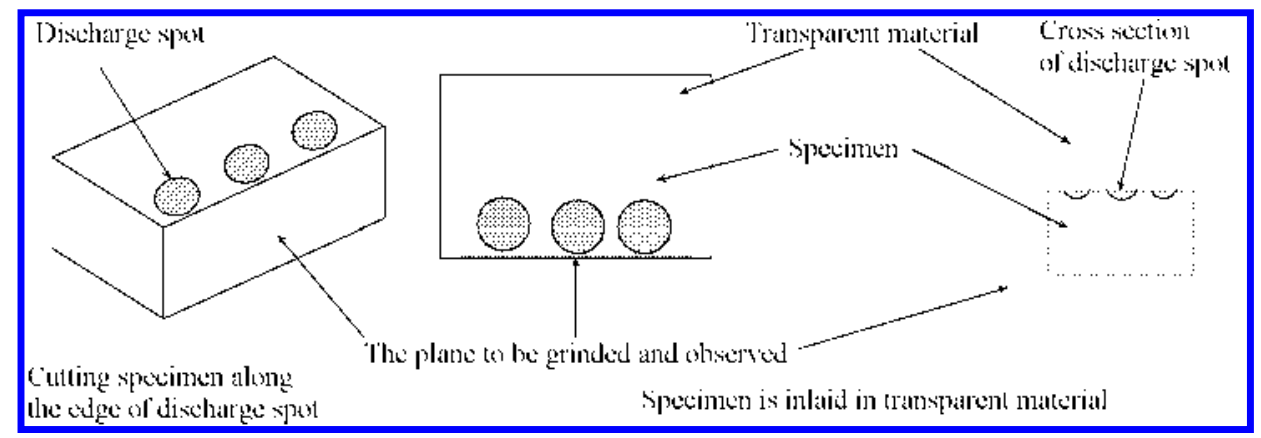

Fig. 5. Specimen making. 


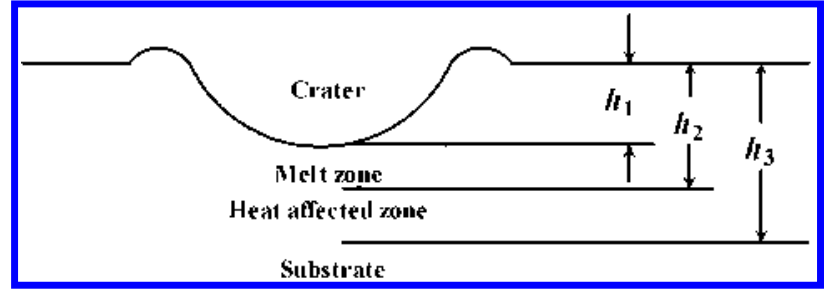

Fig. 6. The presumed profile of LIDT spot cross-section. $h_{1}$ is the depth of crater, $h_{2}$ is the depth of melt zone, $h_{3}$ is the depth of heat affected zone (HAZ).

was corresponding to the diameter of a discharge spot (Fig. 6). In fact, the circumference of discharge spot was not a circle and $h_{3 \max }$ was not exactly corresponding to the maximum diameter of the discharge spot. We measured $h_{3}$ when the plane approached the center of the discharge spot, then grinded out the specimen tens micrometers so that the plane further approached the center and measured $h_{3}$ again. Of course, the plane might pass through the center and $h_{3}$ became smaller. The procedure was repeated three times and the maximum of the three $h_{3}$ was selected as the depth of HAZ of a discharge spot, i.e., $h_{3 \max }$. The average of the three $h_{3 \max }$ was assumed the depth of HAZ of discharge spots which was formed by the same discharge parameters. The metallograph and the measurements were carried out under PolyvarMET microscope.

\section{Results}

\subsection{Typical cross-sections of discharge spots}

Figures 7-10 show typical cross-sections of LIDT spots on steel roll. When the roll is cathode in air, though the spot could be seen, almost no MZ is found and no crater is formed, there is only a thin HAZ, and the surface is smooth (Fig. 7). When the roll is anode in air, the surface has a wave like structure, MZ and HAZ become thick (Fig. 8). When the roll is anode with oil film, a crater appears and it is deep, MZ and HAZ are also thick (Figs. 9 and 10). Figures 11-14 show typical cross-sections of LIDT spot on cast iron. When the roll is in air (cathode or anode), craters are shallow and the surface has a wave like structure, MZ and HAZ are thick (Figs. 11 and 12). When the roll is anode with oil film, the depth of HAZ $h_{3}$ is deeper than that of in air (Figs. 13 and 14). The depth of HAZ $h_{3}$ in oil film with rectangular waveform is deeper than that of triangular waveform (Figs. 10 and 14).

\subsection{The depth of $H A Z$ of multi-crater and single-crater}

It is known there are two types of LIDT crater shape, namely, single-crater and multi-crater, the former is formed if roll is anode with oil film, and the latter

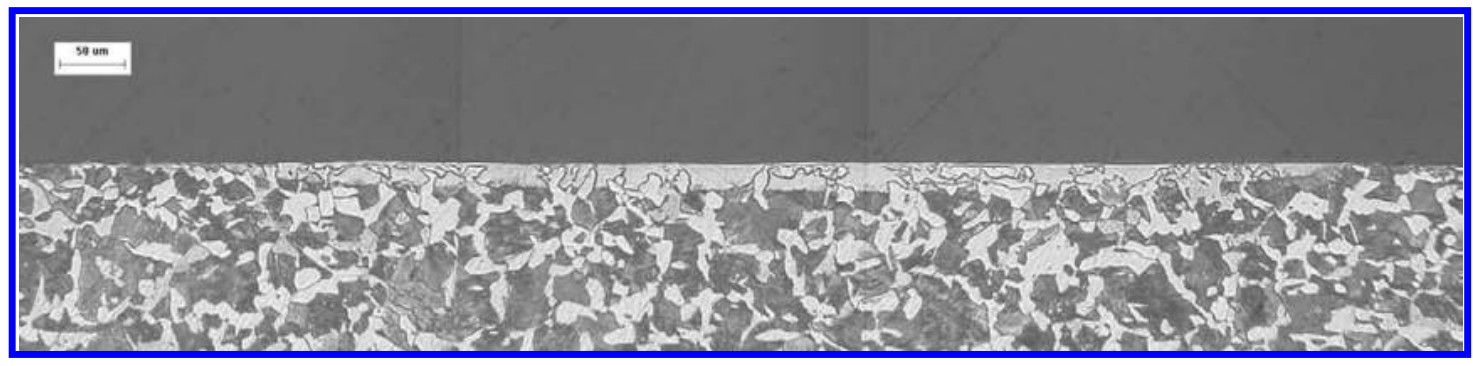

Fig. 7. Cross-section of LIDT spot on steel roll which is used as cathode in air with rectangular waveform.

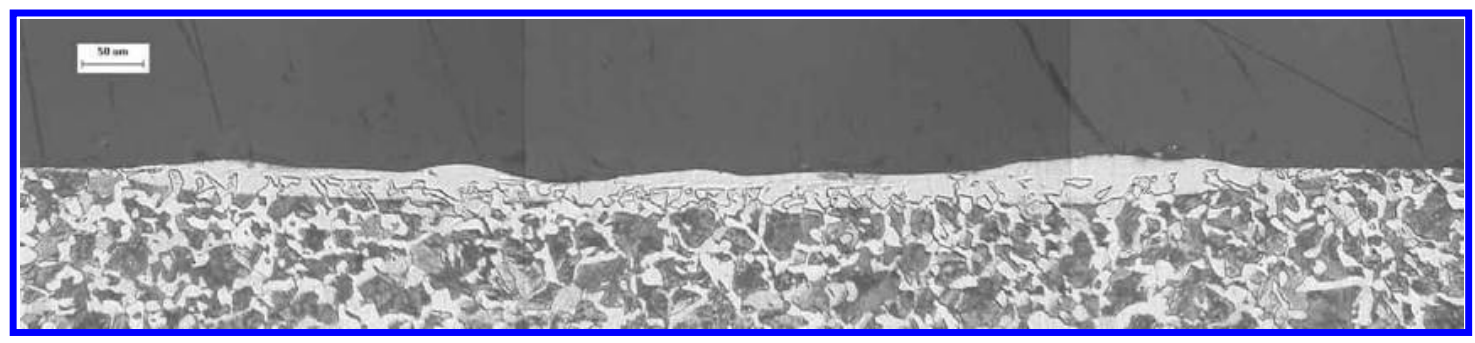

Fig. 8. Cross-section of LIDT spot on steel roll which is used as anode in air with triangular waveform. 


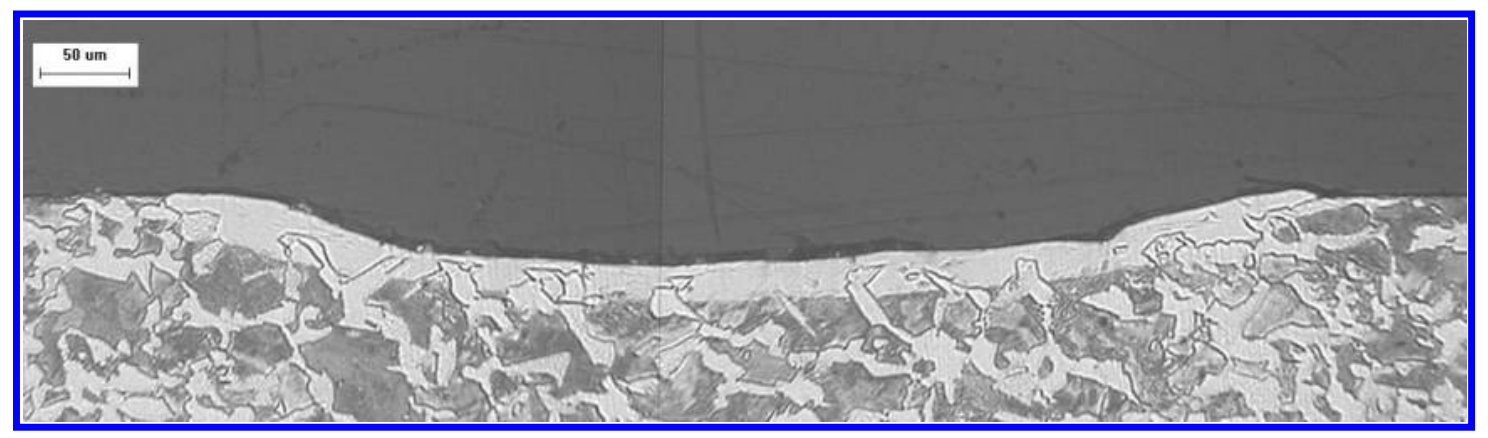

Fig. 9. Cross-section of LIDT spot on steel roll which is used as anode in oil with triangular waveform.

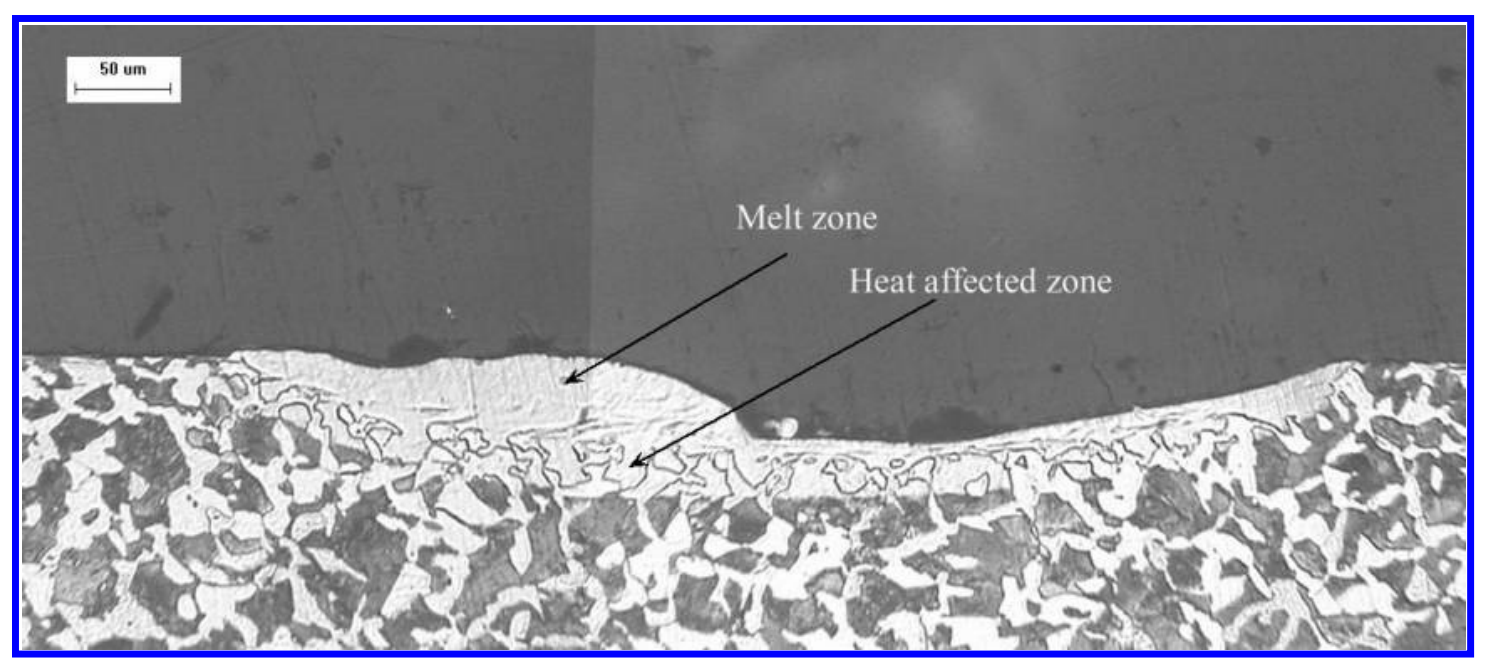

Fig. 10. Cross-section of LIDT spot on steel roll which is used as anode in oil with rectangular waveform.

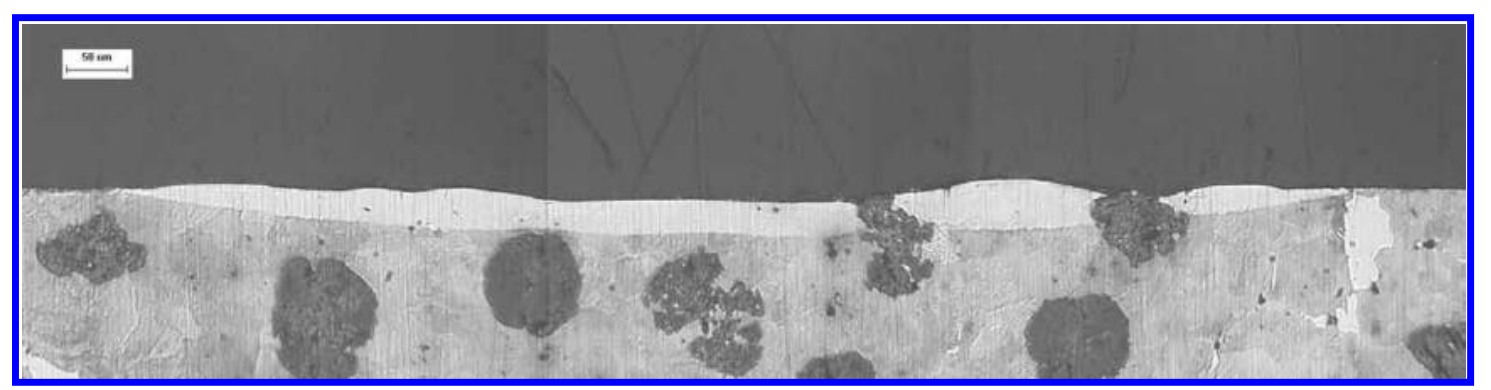

Fig. 11. Cross-section of LIDT spot on cast iron roll which is used as cathode in air with rectangular waveform.

are formed in other discharge conditions. ${ }^{5}$ Current waveforms have little effect on crater morphology. But the depth of HAZ $h_{3}$ with different polarities and waveforms should be investigated.

To compare depth of HAZ $h_{3}$ with different electrical polarities, discharge media and current waveforms, the discharge energy is set as $1 \mathrm{~J}$. Table 1 shows the results. From the table, it is concluded that:
(1) When waveform and polarity are determined, $h_{3}$ with oil film is greater than that of in air.

(2) When polarity and discharge media are determined, $h_{3}$ with rectangular waveform is not smaller than that of triangular waveform.

(3) When discharge media and waveform are determined, $h_{3}$ in anode is deeper than that of in cathode. 


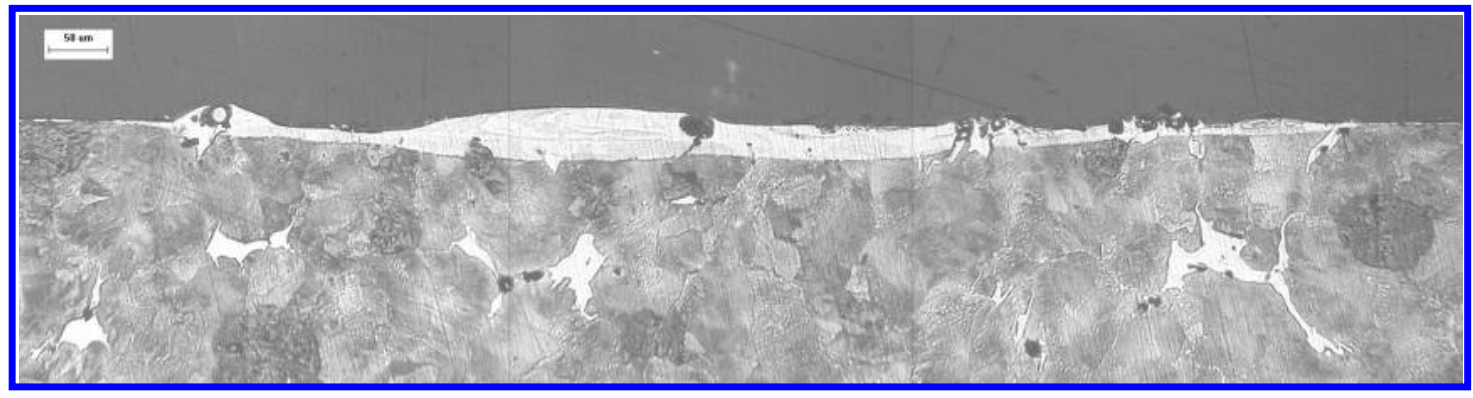

Fig. 12. Cross-section of LIDT spot on cast iron roll which is used as anode in air with triangular waveform.

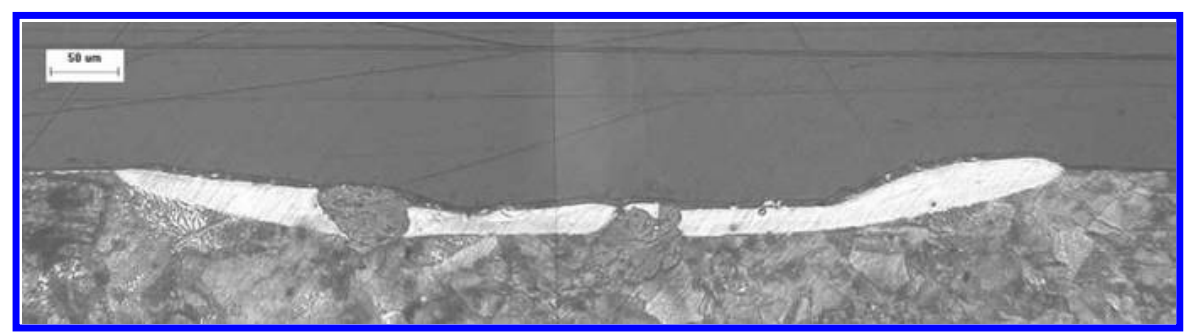

Fig. 13. Cross-section of LIDT spot on cast iron roll which is used as anode in oil with triangular waveform.

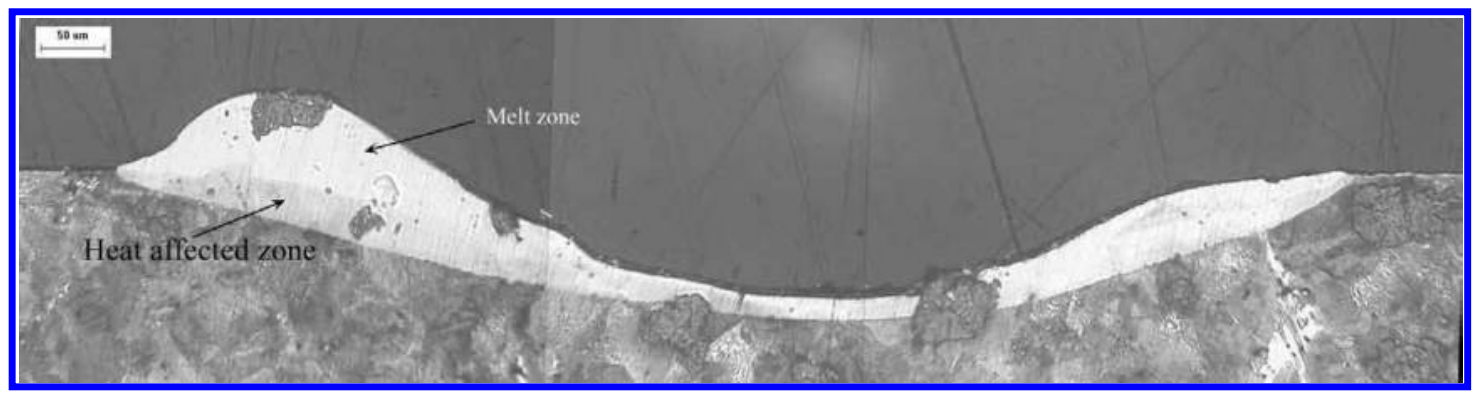

Fig. 14. Cross-section of LIDT spot on cast iron roll which is used as anode in oil with rectangular waveform.

(4) $h_{3}$ of single-crater can reach the most depth among all discharge conditions when discharge energy is the same (see bold line of Table 1).

To investigate rules of $h_{3}$ of single-crater, experiments are carried out on steel and cast iron with anode, oil film and different waveforms. Figure 15 shows $h_{3}$ of single-crater with triangular waveform. Though the discharge energy increases, $h_{3}$ of single-crater on cast iron with triangular waveforms increase only from 52 to $62 \mu \mathrm{m}$. The $h_{3}$ of single-crater on steel has similar results. But $h_{3}$ of single-crater on steel and cast iron with rectangular waveforms increase to nearly double (Fig. 16).

\section{Discussion}

What we concern is which is the main factor to determine depth of HAZ, pulse energy, pulse duration or power density. To calculate average power density of each discharge, crater diameters are measured. It is known that the energy inputted to anode and cathode are about $8 \%$ and $18 \%$ of total discharge energy, respectively. ${ }^{6}$ Therefore, average power density of every pulse discharge on crater can be calculated by the following formula:

$$
\bar{P}=\frac{E \times 8 \%}{\pi\left(\frac{D}{2}\right)^{2} \cdot \tau},
$$


Table 1. Depth of HAZ of LIDT spot on steel and cast iron with different electrical polarities, discharge media and current waveforms at the same electrical pulse energy $(\mu \mathrm{m})$.

\begin{tabular}{llcc}
\hline & \multicolumn{1}{c}{ Discharge conditions } & Steel & Cast iron \\
\hline 1 & Air, anode and rectangular waveform & 52 & 55 \\
$\mathbf{2}$ & Oil, anode and rectangular waveform & $\mathbf{7 8}$ & $\mathbf{7 3}$ \\
3 & Air, anode and triangular waveform & 38 & 38 \\
4 & Oil, anode and triangular waveform & 58 & 52 \\
5 & Air, cathode and rectangular waveform & 20 & 38 \\
6 & Oil, cathode and rectangular waveform & 53 & 50 \\
7 & Air, cathode and triangular waveform & 15 & 28 \\
8 & Oil, cathode and triangular waveform & 53 & 50 \\
\hline
\end{tabular}

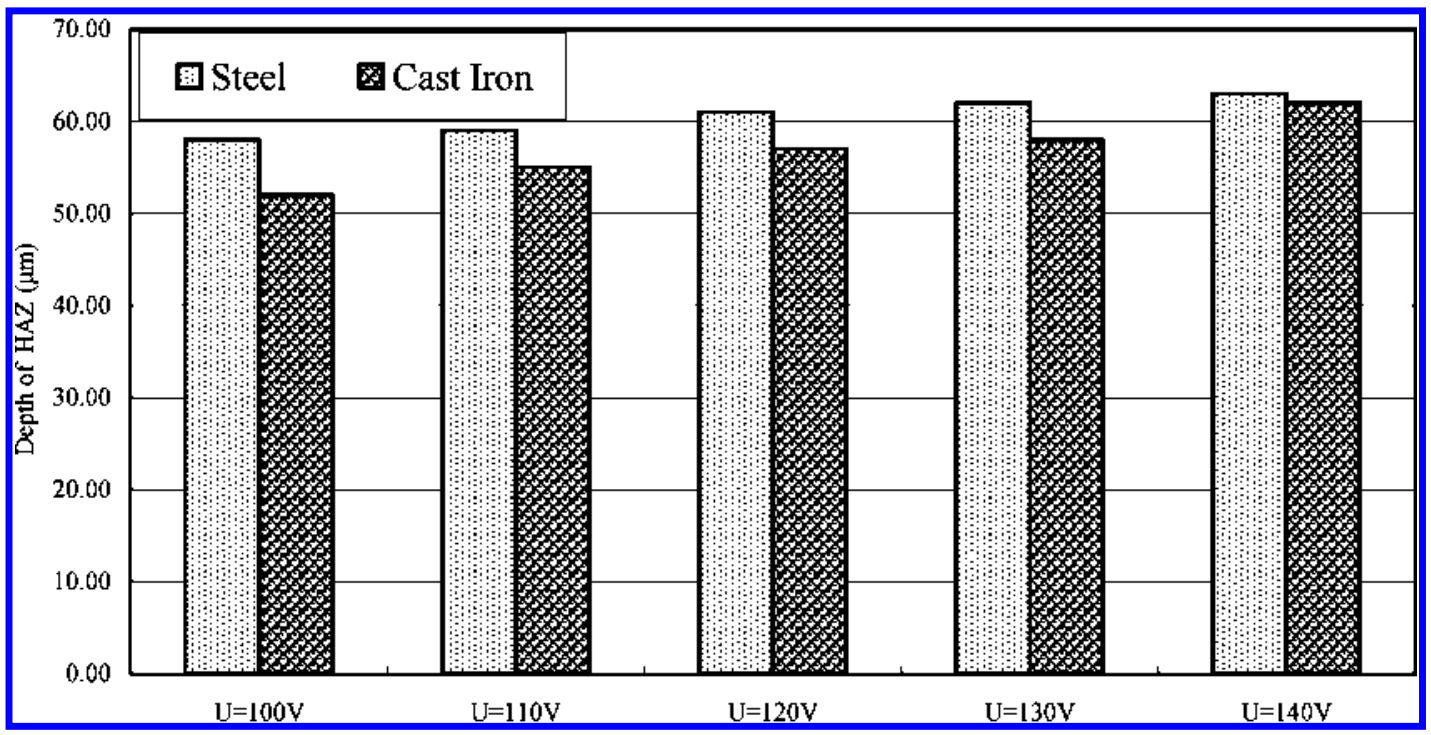

Fig. 15. Depth of HAZ on steel and cast iron with anode, oil film and triangular waveforms.

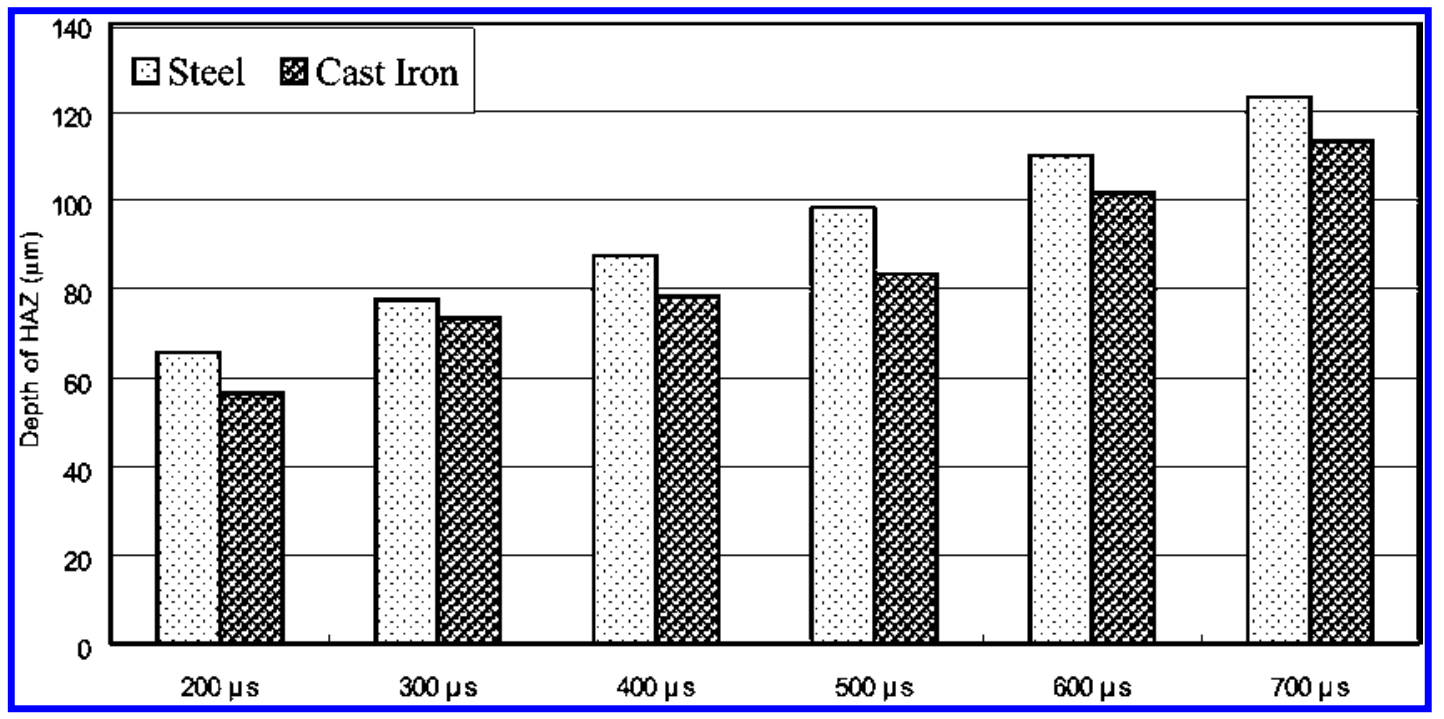

Fig. 16. Depth of HAZ on steel and cast iron with anode, oil film and rectangular waveforms. 
where $\bar{P}, E, D, \tau$ are average power density of each discharge, pulse energy, crater diameter and pulse duration, respectively. The relationship of depth of HAZ $h_{3}$ with energy, pulse duration and average power density are shown in Tables 2, 3, 4 and 5, respectively. In a certain table, with pulse energy increasing, the depth of HAZ $h_{3}$ increases, but the average power density of every discharge decreases slightly. Apparently, the decline power density is due to increasing pulse duration and crater diameter, the latter is correlated with the discharge channel expending. It is also noticed that the order of average power density of rectangular waveforms is about
$10^{4} \mathrm{~W} / \mathrm{cm}^{2}$ lower than that of triangular waveforms $\left(10^{5} \mathrm{~W} / \mathrm{cm}^{2}\right)$, however, the depth of HAZ with rectangular waveforms is greater than that with triangular waveforms in corresponding pulse energy. We understand that triangular waveform has higher current peak than that of rectangular waveform, that results in higher instantaneous power density, but the experimental results is $h_{3}$ of triangular waveform being smaller than that of rectangular waveform. Thus the increase of $h_{3}$ does not depend on average power density.

To investigate effect on $h_{3}$ by pulse energy, we compare the $h_{3}$ with different waveforms in similar

Table 2. Relationship of depth of $\mathrm{HAZ} h_{3}$ with energy, pulse duration and power density of each discharge on steel with triangular waveforms.

\begin{tabular}{ccccc}
\hline$E(\mathrm{~J})$ & Pulse duration $(\mu \mathrm{s})$ & $D(\mu \mathrm{m})$ & $P\left(\mathrm{~W} / \mathrm{cm}^{2}\right)$ & $h_{3}(\mu \mathrm{m})$ \\
\hline 0.99 & 186 & 679 & $1.18 \times 10^{5}$ & 58 \\
1.12 & 195 & 757 & $1.02 \times 10^{5}$ & 59 \\
1.26 & 201 & 795 & $1.01 \times 10^{5}$ & 61 \\
1.42 & 210 & 871 & $9.08 \times 10^{4}$ & 62 \\
1.59 & 225 & 931 & $8.30 \times 10^{4}$ & 63 \\
\hline
\end{tabular}

Table 3. Relationship of depth of HAZ $h_{3}$ with energy, pulse duration and power density of each discharge on cast iron with triangular waveforms.

\begin{tabular}{ccccc}
\hline$E(\mathrm{~J})$ & Pulse duration $(\mu \mathrm{s})$ & $D(\mu \mathrm{m})$ & $P\left(\mathrm{~W} / \mathrm{cm}^{2}\right)$ & $h_{3}(\mu \mathrm{m})$ \\
\hline 1.10 & 174 & 685 & $1.37 \times 10^{5}$ & 52 \\
1.30 & 183 & 744 & $1.31 \times 10^{5}$ & 55 \\
1.45 & 190 & 749 & $1.39 \times 10^{5}$ & 57 \\
1.48 & 196 & 789 & $1.24 \times 10^{5}$ & 58 \\
1.70 & 203 & 819 & $1.27 \times 10^{5}$ & 62 \\
\hline
\end{tabular}

Table 4. Relationship of depth of HAZ $h_{3}$ with energy, pulse duration and power density of each discharge on steel with rectangular waveforms.

\begin{tabular}{ccccc}
\hline$E(\mathrm{~J})$ & Pulse duration $(\mu \mathrm{s})$ & $D(\mu \mathrm{m})$ & $P\left(\mathrm{~W} / \mathrm{cm}^{2}\right)$ & $h_{3}(\mu \mathrm{m})$ \\
\hline 0.66 & 200 & 612 & $8.97 \times 10^{4}$ & 66 \\
$\mathbf{0 . 9 7}$ & $\mathbf{3 0 0}$ & $\mathbf{6 9 4}$ & $\mathbf{6 . 8 4} \times \mathbf{1 0}^{4}$ & $\mathbf{7 8}$ \\
$\mathbf{1 . 2 9}$ & $\mathbf{4 0 0}$ & $\mathbf{7 7 3}$ & $\mathbf{5 . 5 0} \times \mathbf{1 0}^{\mathbf{4}}$ & $\mathbf{8 8}$ \\
$\mathbf{1 . 6 4}$ & $\mathbf{5 0 0}$ & $\mathbf{9 1 6}$ & $\mathbf{3 . 9 8} \times \mathbf{1 0}^{\mathbf{4}}$ & $\mathbf{9 8}$ \\
1.95 & 600 & 1038 & $3.07 \times 10^{4}$ & 110 \\
2.23 & 700 & 1094 & $2.71 \times 10^{4}$ & 123 \\
\hline
\end{tabular}


Table 5. Relationship of depth of HAZ $h_{3}$ with energy, pulse duration and power density of each discharge on cast iron with rectangular waveforms.

\begin{tabular}{ccccc}
\hline$E(\mathrm{~J})$ & Pulse duration $(\mu \mathrm{s})$ & $D(\mu \mathrm{m})$ & $P\left(\mathrm{~W} / \mathrm{cm}^{2}\right)$ & $h_{3}(\mu \mathrm{m})$ \\
\hline 0.64 & 200 & 603 & $8.96 \times 10^{4}$ & 57 \\
$\mathbf{0 . 9 8}$ & $\mathbf{3 0 0}$ & $\mathbf{7 1 8}$ & $\mathbf{6 . 4 5} \times \mathbf{1 0}^{4}$ & $\mathbf{7 3}$ \\
$\mathbf{1 . 3 3}$ & $\mathbf{4 0 0}$ & $\mathbf{8 6 3}$ & $\mathbf{4 . 5 5} \times \mathbf{1 0}^{\mathbf{4}}$ & $\mathbf{7 8}$ \\
$\mathbf{1 . 6 7}$ & $\mathbf{5 0 0}$ & $\mathbf{9 0 6}$ & $\mathbf{4 . 1 4} \times \mathbf{1 0}^{\mathbf{4}}$ & $\mathbf{8 3}$ \\
1.93 & 600 & 950 & $3.63 \times 10^{4}$ & 102 \\
2.25 & 700 & 1009 & $3.22 \times 10^{4}$ & 113 \\
\hline
\end{tabular}

energy. See bold lines on the tables. For example, when the energy is $1.10 \mathrm{~J}$ on cast iron with triangular waveform, the $h_{3}$ is $52 \mu \mathrm{m}$ (Table 3 line 1 ), however the $h_{3}$ reaches $73 \mu \mathrm{m}$ when the energy is $0.98 \mathrm{~J}$ with rectangular waveform (Table 5 line 2). Therefore pulse energy is also not a main factor to determine $h_{3}$.

The only factor to be focused is pulse duration. From the above example, though pulse energies are similar (1.10 J and $0.98 \mathrm{~J}$, respectively), and average power density of the former $\left(1.37 \times 10^{5} \mathrm{~W} / \mathrm{cm}^{2}\right)$ is greater than that of the latter $\left(6.45 \times 10^{4} \mathrm{~W} / \mathrm{cm}^{2}\right)$, pulse duration of the formed $(174 \mu \mathrm{s})$ is smaller than that of the latter $(300 \mu \mathrm{s})$. This phenomenon shows that depth of HAZ depends on pulse duration. In fact, when discharge power density ranges from $10^{4}$ to $10^{5} \mathrm{~W} / \mathrm{cm}^{2}$, it is enough to maintain a melt pool and the depth of HAZ depends on the time of thermal conduction if material and discharge conditions are the same. As long as melt pool maintains, thermal conduction will continue, so the time is approximately equal to the pulse duration. Since thermal conductivity of graphite is lower than that of ferrum, it is found that $h_{3}$ of steel is always greater than that of cast iron in the same waveform in single-crater case.

\section{Conclusions}

Anode, oil film and rectangular waveform are advantages of getting greater depth of HAZ of LIDT spot when discharge energy is the same. The depth of
HAZ mainly depends on pulse duration when LIDT spot is single-crater.

\section{Acknowledgment}

The authors would like to express their gratitude to Natural Science Foundation of China (Grant No. $60278023)$.

\section{References}

1. S. Ujihara, T. Hisatsune, Y. Hishida, M. Imanaka and H. Abe, Application of laser-textured steel sheets for autobody panels, Iron 83 Steel Engineer 68 (1991) $52-56$.

2. K. Nishimura, N. Soeda, S. Ujihara and M. Nagaoka, Development of high image clarity steel sheet by laser texturing, Iron \&s Steel Engineer 68 (1991) 46-51.

3. G. Chen, A new method of texturing surface of roll and its application, Appl. Laser 16 (1996) 155-158 (Chinese).

4. M. Yang, L. Peng and Z. Li, Development of YAG laser texturing technology, Appl. Laser 22 (2002) 316, 323-326 (Chinese).

5. Z. Li, M. Yang, M. Zhong and W. Liu, Investigation on crater morphology by high repetitive rate YAG laser-induced discharge texturing, Surf. Coat. Technol. 200 (2006) 4493-4499.

6. P. T. Eubank, M. R. Patel, M. A. Barrufet and B. Bozkurt, Theoretical models of the electrical discharge machining process. The variable mass, cylindrical plasma model, J. Appl. Phys. 73 (1993) 79007909 . 
This article has been cited by:

1.ZHONGQIANG ZHOU, ZHITONG WANG, MINGJIANG YANG. 2011. THERMAL MODEL FOR LASER-INDUCED DISCHARGE SURFACE STRENGTHENING. Surface Review and Letters 18:06, 289-296. [Abstract] [References] [PDF] [PDF Plus] 